\title{
Plasma wakefield excitation by incoherent laser pulses: a path towards high-average power laser-plasma accelerators
}

\author{
C. Benedetti, C. B. Schroeder, E. Esarey, and W. P. Leemans \\ Lawrence Berkeley National Laboratory, Berkeley, California 94720, USA
}

(Submitted to Nature Publishing Group, 2013) 
In a laser plasma accelerator (LPA), a short and intense laser pulse propagating in a plasma drives a wakefield (a plasma wave with a relativistic phase velocity) that can sustain extremely large electric fields, enabling compact accelerating structures $[1-4]$. Potential LPA applications include compact radiation sources $[5-8]$ and high energy linear colliders [9-11]. We propose and study plasma wave excitation by an incoherent combination of a large number of low energy laser pulses (i.e., without constraining the pulse phases). We show that, in spite of the incoherent nature of electromagnetic fields within the volume occupied by the pulses, the excited wakefield is regular and its amplitude is comparable or equal to that obtained using a single, coherent pulse with the same energy. These results provide a path to the next generation of LPA-based applications, where incoherently combined multiple pulses may enable high repetition rate, high average power LPAs.

The plasma wave excited by a laser pulse propagating in an underdense plasma is the result of the gradient in laser field energy density providing a force (i.e., the ponderomotive force) that creates a space charge separation between the plasma electrons and the neutralizing ions. For a resonant laser pulse, i.e., with a duration $T_{0} \sim \lambda_{p} / c$, where $\lambda_{p}=2 \pi / k_{p}=2 \pi c / \omega_{p}$ is the plasma wavelength, and $\omega_{p}=\left(4 \pi n_{0} e^{2} / m\right)^{1 / 2}$ is the electron plasma frequency for a plasma with density $n_{0}$ ( $c$ is the speed of light in vacuum, $m$ and $e$ are, respectively, the electron mass and charge), with a relativistic intensity, i.e., with a peak normalized vector potential $a_{0}=e A_{0} / m c^{2} \sim 1\left(A_{0}\right.$ is the peak amplitude of the laser vector potential), the amplitude of the accelerating field is of order $E_{0}=m c \omega_{p} / e$, or $E_{0}[\mathrm{~V} / \mathrm{m}] \simeq 96 \sqrt{n_{0}\left[\mathrm{~cm}^{-3}\right]}$. For example, $n_{0} \sim 10^{17} \mathrm{~cm}^{-3}$ gives $E_{0} \simeq 30 \mathrm{GV} / \mathrm{m}$, which is three orders of magnitude higher than in conventional accelerators.

Applications for LPAs that produce electron beams with energies 1-10 GeV include free electron lasers in the x-ray regime [5 8] and accelerator modules for linear colliders [9 11$]$. For example, a conceptual design for a $1 \mathrm{TeV}$ center-of-mass electron-positron LPA-based linear collider is presented in Ref. 10. Both the electron and positron arms of the collider require $50 \mathrm{LPA}$ stages at $10 \mathrm{GeV}$ with a length of $\sim 1 \mathrm{~m}$ and a density $n_{0} \sim 10^{17} \mathrm{~cm}^{-3}$. Each LPA stage is powered by a laser pulse with tens of $\mathrm{J}$ of energy with duration $T_{0} \lesssim 100$ 
fs and an intensity such that $a_{0} \sim 1$, which is achievable with present laser technology. The required laser repetition rate, however, as dictated by luminosity requirements, is $f_{\text {rep }} \sim 10$ $\mathrm{kHz}$ (an average power of hundreds of $\mathrm{kW}$ ), which is orders of magnitude beyond present technology.

To date, LPAs are typically driven by solid-state (e.g., Ti:sapphire) lasers that are limited to an average power $\sim 100 \mathrm{~W}$. For example, the Berkeley Lab Laser Accelerator (BELLA) laser delivers $40 \mathrm{~J}$ pulses on target at $1 \mathrm{~Hz}$ [12]. Since virtually all applications of LPA will benefit greatly from higher repetition rates, it is essential that high average power laser technology continues to be developed.

Several laser technologies are being developed to provide systems with high average power and high efficiency [2, 13]. For example, in Refs. 13 and 14, a scheme is presented were a large number of diode-pumped fiber systems, delivering pulses with $\sim \mathrm{mJ}$ energy at $\mathrm{kHz}$ repetition rate, are combined in such a way the relative phases of the output beams are controlled so they constructively interfere (coherent combination) and produce a single, high power output beam with high efficiency. Coherent combination of a large number of fiber lasers (e.g., $\sim 10^{4}$ fibers for a total pulse energy of 10's of J) for an LPA is extremely challenging, requiring short pulse beams (duration $<$ ps) that are matched in phase, time and space. To date, the coherent combination of an array of 64 (continuous wave) beams from fiber amplifiers has been demonstrated [15]. The coherent combination of a small number of femtoseconds pulses has also been achieved [16, 17].

As is shown below, an LPA does not require a fully coherent drive laser pulse. This is true because the LPA wakefield is excited by the ponderomotive force (i.e., the gradient in the electromagnetic energy density), along with the fact that the plasma responds on the time scale $\lambda_{p} / c$. Large amplitude wakefield excitation requires sufficient electromagnetic energy within a given volume, typically of the order of $\sim \lambda_{p}^{3}$. Since the wakefield response behind the driver depends on the time-integrated behavior of the electromagnetic energy density of the driver over $\lambda_{p} / c$, it is insensitive to time structure in the driver on time scales $\ll \lambda_{p} / c$, which allows for the use of incoherently combined laser pulses as the driver. Theoretically, this can be easily demonstrated in the linear $\left(a^{2}<1\right)$ wakefield regime in which the amplitude of the electric field of the wake is small $\left(|\mathbf{E}| / E_{0}<1\right)$. In the linear regime, the wake electric field $\mathbf{E}$ is given by [1]

$$
\left(\partial^{2} / \partial t^{2}+\omega_{p}^{2}\right) \mathbf{E} / E_{0}=-\left(c \omega_{p} / 2\right) \nabla a^{2},
$$


with the solution

$$
\mathbf{E} / E_{0}=-(c / 2) \int_{0}^{t} d t^{\prime} \sin \left[\omega_{p}\left(t-t^{\prime}\right)\right] \nabla a^{2}\left(t^{\prime}\right),
$$

This Green function solution averages out the small scale time structure in the ponderomotive force. Hence, in effect, the wakefield is given by

$$
\left(\partial^{2} / \partial t^{2}+\omega_{p}^{2}\right) \mathbf{E} / E_{0} \simeq-\left(c \omega_{p} / 2\right) \nabla\left\langle a^{2}\right\rangle
$$

where the angular brackets represent a time average over scales small compared to $\lambda_{p} / c$.

Owing to the time average process characterizing the wake excitation, we show that multiple, low-energy, incoherently combined laser pulses can deposit sufficient field energy in the plasma to ponderomotively drive a large wakefield. We show that no phase control in the combination of multiple laser pulses is required for LPAs. We find that, under certain conditions, the wake generated by an incoherent combination of pulses is regular behind the driver and its amplitude is comparable, or equal, to the one obtained by using a single coherent pulse with the same energy. We expect that the fundamental requirements to achieve incoherent combination are more relaxed compared to coherent combination. Hence, incoherent combination may provide an alternative and technically simpler path to the realization of high repetition rate and high average power LPAs.

To illustrate the physics of wake generation by incoherently combining multiple laser pulses, we present two examples of incoherent combination schemes, namely spectral combination and mosaic of beamlets. In the first example we consider $N$ laser pulses with the same length and spot size, and different (random) phases, propagating (along $z$ direction) in a parabolic plasma channel. The laser spot size is the one ensuring matched propagation in the channel. The pulses have different wavelengths and they are spectrally separated (i.e., the power spectra of the pulses do not overlap with each other) and so the total energy of the incoherent combination equals the sum of the energies of the pulses. From an experimental point of view, the condition of spectral separation allows the overlap of the different pulses by using a dispersive optical system like a sequence of dichroic mirrors, a grating, or a prism [18]. In Fig. 17 we show (red line) the on-axis lineout of the driver energy density generated by spectrally combining 48 pulses. The pulses have the same energy (and intensity), and their centroids are overlapped. For each frequency in the combination we accommodate two laser pulses with orthogonal polarizations (polarization multiplexing). The black dashed line in the same panel is the laser energy density for a single (circularly polarized) coherent pulse 

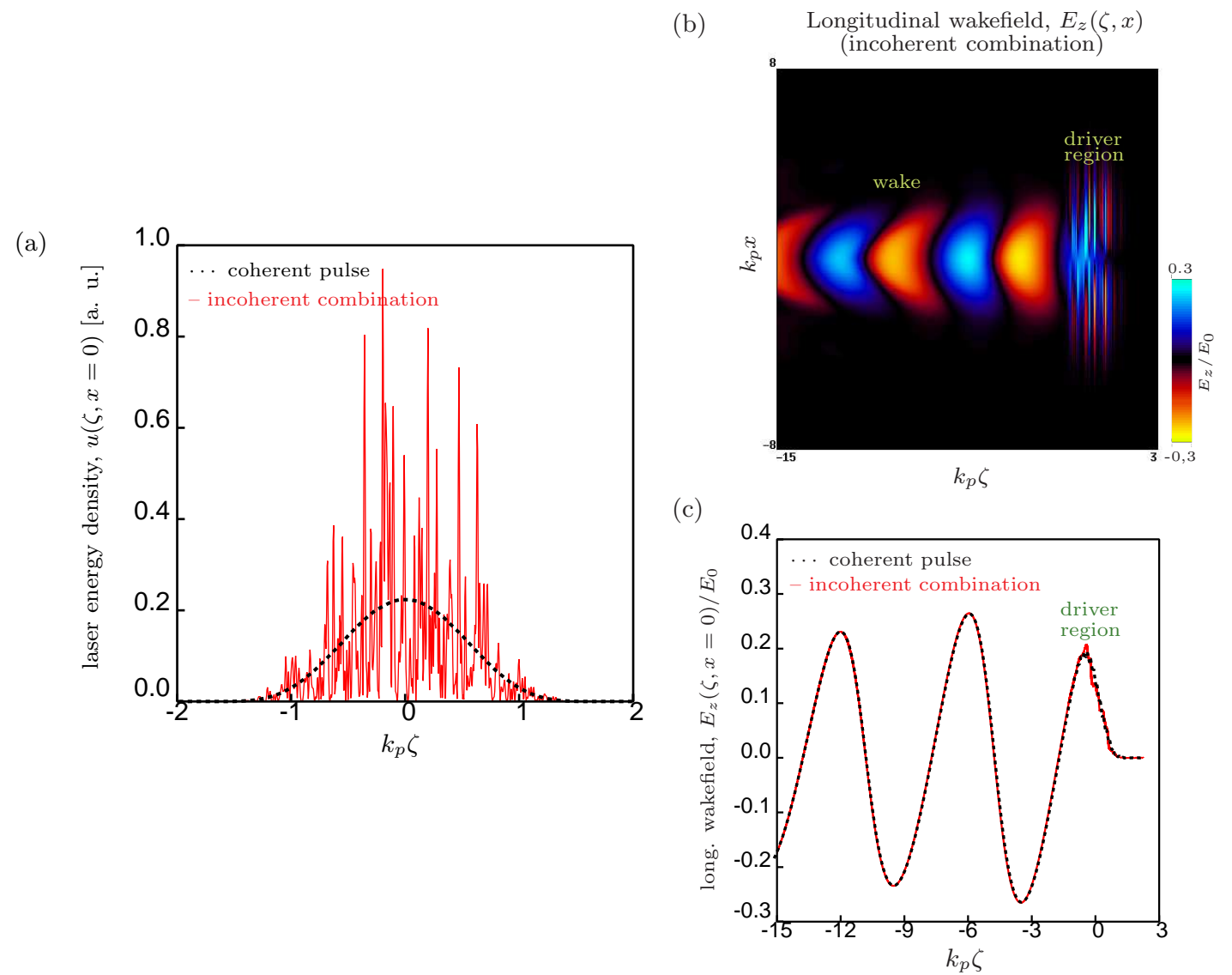

\section{FIG. 1. Laser energy density and wakefield generated by a wavelength combining}

scheme. a, On-axis lineout (red line) of the electromagnetic energy density obtained overlapping 48 spectrally separated laser pulses (including polarization multiplexing). The field of each pulses has a cosine-squared longitudinal profile with a FWHM duration of $30 \mathrm{fs}$, and a Gaussian transverse profile with a spot size of $12 \mu \mathrm{m}$. All the pulses have the same energy. The minimum and maximum laser wavelengths of the pulses are $\lambda_{0, \min }=0.2 \mu \mathrm{m}\left(a_{0}\left(\lambda_{0, \min }\right)=0.03\right)$, and $\lambda_{0, \max }=2.7 \mu \mathrm{m}$ $\left(a_{0}\left(\lambda_{0, \max }\right)=0.47\right)$. The laser beams propagate (matched propagation) in a parabolic plasma channel with an on-axis density $n_{0}=10^{18} \mathrm{~cm}^{-3}$. The black dashed line is the electromagnetic energy density for a single, circularly polarized, coherent, laser pulse with an energy equal to that of the incoherently combined pulses, and an intensity such that $a_{0, \text { coherent }}=1$ for $\lambda_{0}=0.8 \mu \mathrm{m}$. $\mathbf{b}$, Map of the longitudinal wakefield, $E_{z}(\zeta, x)$, generated by the incoherent combination. c, On-axis lineout of the longitudinal wakefield for the incoherent combination (red line) and for the coherent pulse (black dashed line). 
with the same energy (see Fig. 1 caption for the laser and plasma parameters). In Fig. 1b we show a map, obtained with the PIC code ALaDyn [19, 20], of the longitudinal wakefield, $E_{z}(\zeta, x), \zeta=z-c t$ being the longitudinal coordinate relative to the laser driver and $x$ the transverse coordinate, generated by the incoherent combination. Finally, Fig. 11. shows the on-axis lineout of $E_{z}$ for the incoherent combination (red line) and for the circularly polarized laser pulse (black dashed line). We notice, from both Fig. 1b and Fig. 1k, that the wakefield from incoherent combination shows an incoherent pattern within the driver region, namely for $\left|k_{p} \zeta\right| \lesssim 2$. However, behind the driver region $\left(k_{p} \zeta \lesssim-2\right)$, the wakefield is regular and its amplitude equals the one of the single coherent pulse with the same energy.

In the second example we consider a collection of short and narrow laser beamlets placed side-by-side, both longitudinally and transversally, tiling a prescribed volume. Each pulse has high (relativistic) peak intensity but low energy owing to the limited spatial extent of the beamlets. The beamlets, whose centroids are arranged into an uniform spatial grid, have the same length $\left(\ell_{0}\right)$, width $\left(d_{0}\right)$, wavelength $\left(\lambda_{0}\right)$ and intensity $\left(a_{0}\right)$, and different (random) phases. For each centroid location we can accommodate two beamlets with orthogonal polarizations (polarization multiplexing). The laser field of each beamlet vanishes at a distance from the centroid larger than $\ell_{0} / 2$, longitudinally, and $d_{0} / 2$, transversally, and the fields of beamlets with the same polarization do not overlap. As a consequence, energy is additive (i.e., the total energy is the sum of the energy of the single beamlets). For simplicity, in this example we will restrict the discussion to two-dimensional (2D) Cartesian geometry. The generalization to the three-dimensional case is straightforward. The guiding of the incoherent combination of pulses over distances much longer compared to the Rayleigh length of the single laser beamlets, namely $Z_{R} \sim \pi d_{0}^{2} / \lambda_{0}$, can be achieved by a plasma channel with, transversally, a constant density over the domain occupied by the beamlets, followed by steep plasma walls able to reflect and contain radiation from diffracting beamlets. Because of multiple reflections, and interference between the fields of different beamlets, we expect the total electromagnetic radiation driving the wake to have a complex pattern. In Fig. 2 we show the evolution of the rms transverse size of the energy distribution, $\sigma_{x}(s)$, $s=c t$ being the propagation distance, for a combination of 208 beamlets initial arranged in an array of $13 \times 16$ (see Fig. 2 caption for the laser and plasma parameters). We see that the laser energy from the combination is well guided over distances significantly longer than the Rayleigh length of the beamlets. The two insets show snapshots of the laser energy 


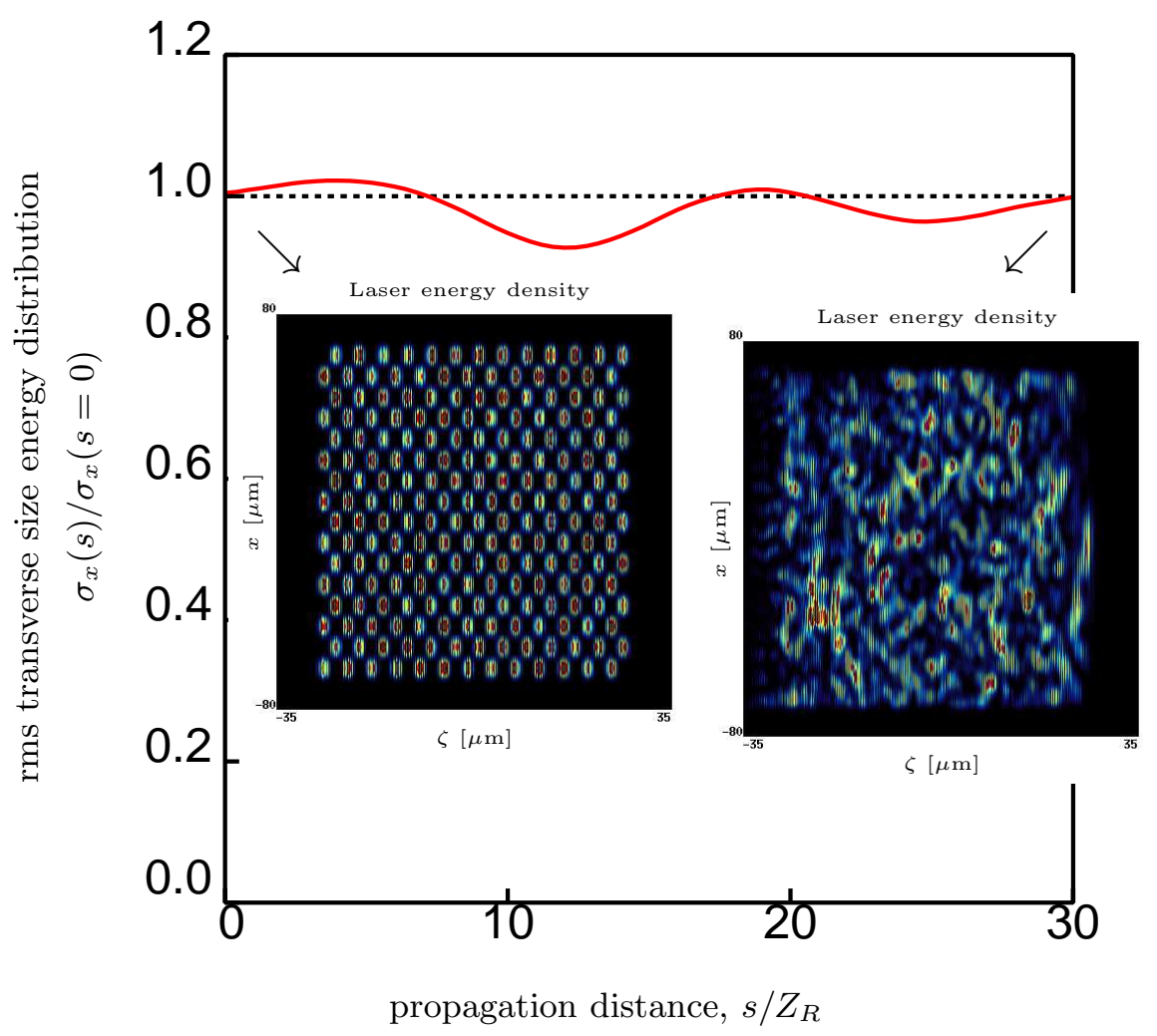

FIG. 2. Guiding of a mosaic of beamlets. Evolution of the rms transverse size of the energy distribution, $\sigma_{x}(s)$, for a combinations of $208=13 \times 16$ identical beamlets (the field of each beamlet has a cosine-squared dependence but longitudinally and transversally) with $a_{0}=1.5, \ell_{0}=4 \mu \mathrm{m}$ (total pulse length), $d_{0}=15 \mu \mathrm{m}$ (total pulse width), $\lambda_{0}=0.8 \mu \mathrm{m}$. The background plasma density is $n_{0}=0.9 \cdot 10^{17} \mathrm{~cm}^{-3}$. The beamlets are tiling a $2 \mathrm{D}$ domain $55 \mu \mathrm{m} \simeq \lambda_{p} / 2$ long and $144 \mu \mathrm{m}$ wide. The two insets show time snapshots of the laser energy density at the beginning of the simulation (left), and after some propagation distance in the plasma (right), where the laser field exhibits an incoherent pattern.

density at the beginning of the simulation (initial beamlets configuration), and after some propagation distance in the plasma, where the laser field exhibits a clear incoherent pattern.

The wakefield generated by the mosaic of incoherent beamlets is presented in Fig. 3. The laser and plasma parameters are the same as in Fig. 2. In Fig. 3a we show a 2D map of the longitudinal wakefield generated by the incoherent combination after a propagation 

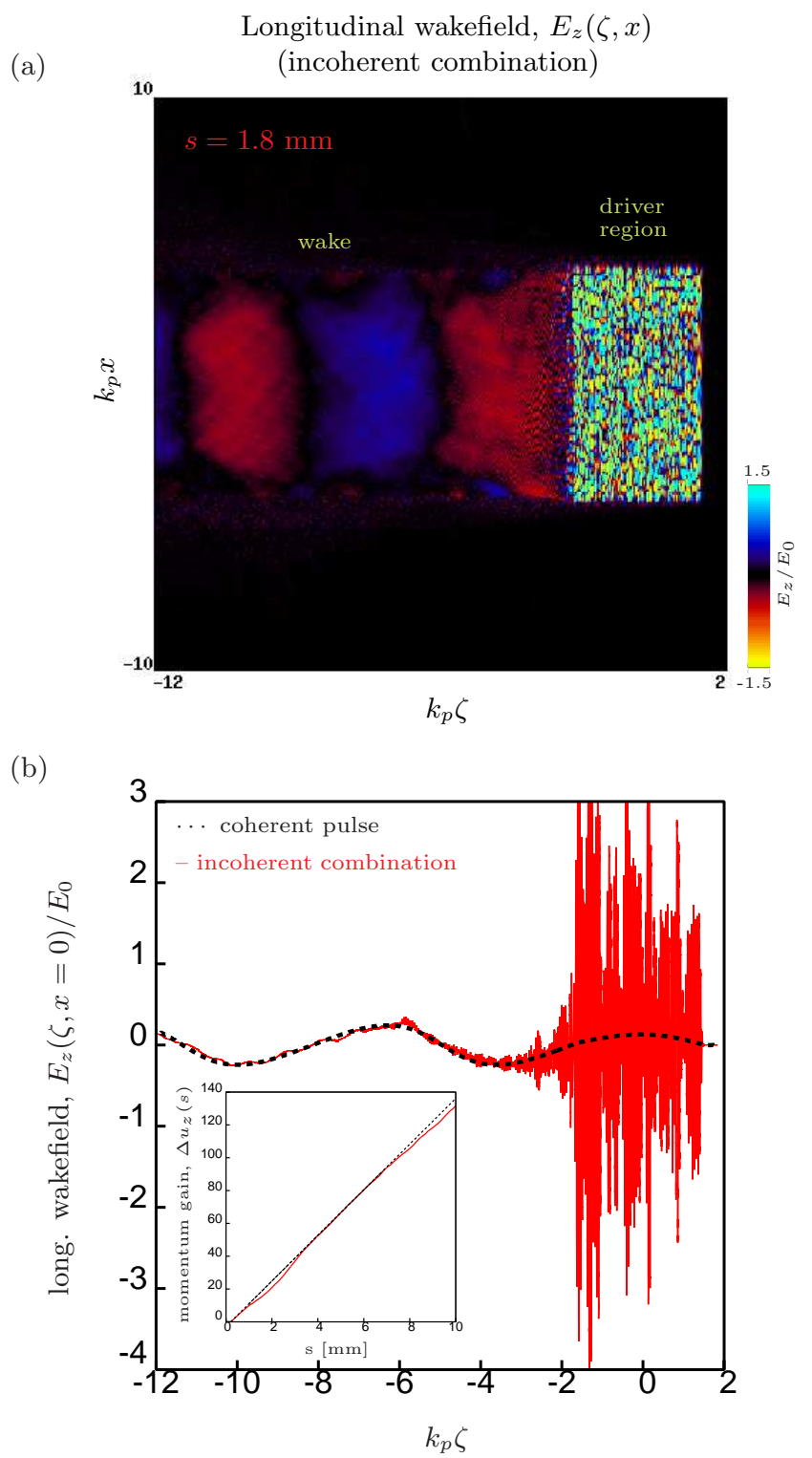

FIG. 3. Wakefield generated by a mosaic of beamlets. a, Map of the longitudinal wakefield, $E_{z}(\zeta, x)$, generated by the incoherent combination of 208 beamlets (same parameters as in Fig. 2) after a propagation distance $s=1.8 \mathrm{~mm}$ in the plasma. $\mathbf{b}$, On-axis lineout of the longitudinal wakefield for the incoherent combination (red line) and for a single coherent pulse with $a_{0, \text { coherent }}=$ 0.8 and same length and rms spot size as the incoherent combination (black dashed line). This inset shows the integrated momentum gain, $\Delta u_{z}(\zeta, s) \simeq-\left(e / m c^{2}\right) \int_{0}^{s} E_{z}\left(\zeta, x=0, s^{\prime}\right) d s^{\prime}$, for a relativistic particle initially located in $k_{p} \zeta \simeq-10$ (maximum accelerating field). The black and red lines refer, respectively, to the momentum gain in the coherent and incoherent case.

distance $s=1.8 \mathrm{~mm}$. In Fig. 3b we show the on-axis lineout of the longitudinal wakefield 
for the incoherent combination (red line) and for a single coherent pulse (uniform transverse intensity profile) with an intensity such that $a_{0 \text {,coherent }}=0.8$, and with the same length and rms spot size as the incoherent combination (black dashed line). We notice that, behind the driver region, the wake from incoherent combination is regular and its amplitude is the same as the one from a single coherent pulse. The noisy field structure observed in the lineout of $E_{z}$, due to multiple reflections from walls and interference of beamlets, does not affect the energy gain of relativistic particles accelerated in the wakefield. This is shown in the inset of Fig. 3] $\mathrm{b}$, where we compute the integrated momentum gain as a function of the propagation distance for a relativistic particle initially located in $k_{p} \zeta \simeq-10$ (maximum accelerating field). The black and red lines in the inset refer, respectively, to the momentum gain in the coherent and incoherent case. No significant difference is observed in the momentum gain in the two cases ( $\sim 2 \%$ difference in the energy gain after $10 \mathrm{~mm}$ propagation). The total energy of the combination of pulses exceeds the one of the coherent pulse by $\sim 10 \%$. This (moderate) loss in the efficiency of the combination can be partially ascribed to the fact that the varying transverse intensity profile within each beamlet results in an effective less-than-unity fill factor.

In summary, we have shown that it is possible to efficiently excite a wakefield for LPA applications with an incoherent combination of multiple, low-energy, laser pulses. Since no phase control is required, we expect that the fundamental requirements to achieve incoherent combination are more relaxed compared to coherent combination, thereby enabling a technologically simpler path for design of high-average power, high-repetition rate LPA applications.

[1] Esarey, E., Schroeder, C. B. \& Leemans, W. P. Physics of laser-driven plasma-based electron accelerators.. Rev. Mod. Phys. 81, 1229-1285 (2009).

[2] Hooker, S. Developments in laser-driver plasma accelerators. Nature Photon. 7, 775-782 (2013).

[3] Leemans, W. P. et al. GeV electron beams from a centimetre-scale accelerator. Nature Phys. 2, 696-699 (2006).

[4] Wang, X. et al. Quasi-monoenergetic laser-plasma acceleration of electrons to $2 \mathrm{GeV}$. Nat 
Commun. 4, 1988 (2013).

[5] Leemans, W. P. et al. Observation of terahertz emission from a laser-plasma accelerated electron bunch crossing a plasma-vacuum boundary. Phys. Rev. Lett. 91, 074802 (2003).

[6] Fuchs, M. et al. Laser-driven soft-x-ray undulator source. Nature Phys. 5, 826-829 (2009).

[7] Maier, A. R. et al. Demonstration Scheme for a Laser-Plasma-Driven Free-Electron Laser. Phys. Rev. X 2, 031019 (2012).

[8] Huang, Z., Ding, Y., \& Schroeder, C. B. Compact X-ray free-electron laser from a laser-plasma accelerator using a transverse-gradient undulator. Phys. Rev. Lett. 109, 204801 (2012).

[9] Leemans, W. P. \& Esarey, E. Laser-driven plasma-wave electron accelerators. Phys. Today 62, 44-49 (2009).

[10] Schroeder, C. B., Esarey, E., Geddes, C. G. R., Benedetti, C. \& Leemans, W. P. Physics considerations for laser-plasma linear colliders. Phys. Rev. ST Accel. Beams 13, 101301 (2010); Schroeder, C. B., Esarey, E., \& Leemans, W. P. Phys. Rev. ST Accel. Beams 15, 051301 (2010).

[11] Nakajima, K. et al. Operating plasma density issues on large-scale laser-plasma accelerators toward high-energy frontier. Phys. Rev. ST Accel. Beams 14, 091301 (2011).

[12] W.P. Leemans et al., Particle Accelerator Conference Proc. (2013).

[13] Mourou, G., Brocklesby, B., Tajima, T. \& Limpert, J. The future is fibre accelerators. Nature Photon. 7, 258-261 (2011).

[14] Mourou, G. A., Hulin, D. \& Galvanauskas, A. The road to high peak power and high average power lasers: Coherent-Amplification-Network (CAN). AIP Conf. Proc. 827, 152-163 (2006).

[15] Bourderionnet, J., Bellanger, C., Primot, J. \& Brignon, A. Collective coherent phase combining of 64 fibers. Opt. Express 19, 170530-17058 (2011).

[16] Daniault, L. et al. Passive coherent beam combining of two femtosecond fiber chirped-pulse amplifiers. Opt. Lett. 36, 4023-4025 (2011).

[17] Klenke, A. et al. Coherently-combined two channel femtosecond fiber CPA system producing 3 mJ pulse energy. Opt. Express 19, 24280-24285 (2011).

[18] Fan, T. Y. Laser beam combining for high-power, high-radiance sources. IEEE Journal of Selected topics in quantum electronics 11, 567-577 (2005).

[19] Benedetti, C., Sgattoni, A., Turchetti, G. \& Londrillo, P. ALaDyn: A high-accuracy PIC code for the Maxwell-Vlasov equations. IEEE Transactions on plasma science 36, 1790-1798 
(2008).

[20] Benedetti, C. et al. PIC simulations of the production of high-quality electron beams via laser-plasma interaction. Nucl. Instr. and Meth. A 608, S94-S98 (2009).

\section{ACKNOWLEDGMENTS}

This work was first presented by C. Benedetti at the APS Division of Plasma Physics Meeting, Denver, CO, November 12, 2013, and subsequently submitted for publication.

This work was supported by the Director, Office of Science, Office of High Energy Physics, of the U.S. DOE under Contract No. DE-AC02-05CH11231, and used the computational facilities (Hopper, Edison) at the National Energy Research Scientific Computing Center (NERSC). We would like to thank A. Galvanauskas for useful discussions and suggestions. 